\title{
Sistema de Internet das Coisas para Detecção de Incêndios Florestais
}

\author{
Sílvia Albuquerque ${ }^{1}$, Daniel Fonseca ${ }^{1}$, Raphael Luiz Lima ${ }^{1}$, \\ Anolan Milanés ${ }^{1}$, Isabela Cristina Rocha ${ }^{1}$, Ana Maria Santos ${ }^{1}$, \\ Gabriel Silva $^{1}$, Fadoa Vieira ${ }^{1}$ \\ ${ }^{1}$ Departamento de Computação do CEFET-MG \\ Av. Amazonas 7675, Nova Gameleira, Belo Horizonte, MG, Brasil \\ \{anolan, silviacalmon\}@cefetmg.br
}

\begin{abstract}
This article presents the results of experimental tests of commercial sensors and communication modules for the development of a low-cost Internet of Things (IoT) system prototype for early forest fires detection based on WiFi and LoRa standards. The system is composed of three parts: (1) sensor nodes, which measure temperature, humidity and concentration of flammable gases in the environment; (2) a gateway, which receives data read by nodes and sends it to the Internet; and (3) a Web system to display the measurements of the sensors. The sensor tests indicated that they only capture information from the close environment, making it difficult to monitor large areas. Tests of the communication modules in a cerrado vegetation environment showed data transmissions at distances of approximately 200 meters for the Wi-Fi standard and 500 meters for the LoRa, confirming that it has a greater range than the Wi-Fi, but much less than the expected kilometers range acording to standard's specifications.
\end{abstract}

Resumo. Este artigo apresenta o resultado dos testes de sensores e de módulos de comunicação comerciais efetuados como parte do desenvolvimento de um protótipo de sistema de Internet das Coisas (IoT) de baixo custo para a detecção precoce de incêndios florestais. O sistema é composto por três partes: (1) nós sensores, que medem a temperatura, a umidade e a concentração de gases inflamáveis do ambiente; (2) um gateway, que recebe os dados lidos pelos nós e os envia para a Internet; e (3) um sistema Web para mostrar as medições dos sensores. Os testes dos sensores indicaram que estes captam apenas informações do ambiente próximo, dificultando o monitoramento de áreas extensas. Os testes dos módulos de comunicação em um ambiente de vegetação de cerrado mostraram transmissões de dados a distâncias de aproximadamente 200 metros para o padrão Wi-Fi e 500 metros para o LoRa, confirmando que este possui um maior alcance do que o Wi-Fi, porém não alcança a distância de alguns quilômetros, esperada segundo as especificações do padrão. 


\section{Introdução e Contextualização}

Os incêndios florestais são um dos principais fenômenos recorrentes que provocam a destruição de áreas naturais e causam um imenso dano a fauna e a flora desses locais. Frequentemente, provocam também a destruição de construções próximas as regiões afetadas e a perda de vidas humanas. Condições como clima quente, estações muito secas e grandes períodos de estiagem, contribuem à maior intensidade e frequência dos incêndios florestais [Borunda 2019]. Por exemplo, o incêndio ocorrido em novembro de 2018 na cidade de Paradise, na Califórnia, provocou a destruição de mais de $500 \mathrm{mil} \mathrm{km}^{2}$ e a morte de 42 pessoas [G1 2018].

Além dos fatores já citados, outro que dificulta a prevenção e a detecção de incêndios florestais é a pequena quantidade de pessoas responsáveis por áreas extensas. Assim, surge o problema de monitoramento e a detecção precoce do fogo, que se ocorrer em tempo hábil pode reduzir significativamente o impacto nessas áreas, bem como o custo e tempo necessários para combater o incêndio [Alkhatib 2013].

Assim, considerando as características do fogo é possível o uso de diversas abordagens para a detecção de incêndios florestais, como: a utilização de câmeras de alta resolução com imagens ópticas, infravermelhas ou térmicas; som acústico de rádio com forma de inferir o fluxo meteorológico ou os perfis de temperatura nas áreas florestais e rede de sensores [Zhang et al. 2016].

Se comparada às outras soluções citadas, sistemas baseados em sensores e Internet das Coisas (Internet of Things - IoT) possuem uma das melhores relações custo-benefício [Alkhatib 2014]. Esses sistemas possuem um menor tempo entre o início do incêndio e o alerta aos responsáveis, além de uma maior manutenibilidade [Alkhatib 2013].

Durante um incêndio há a liberação de gases na atmosfera e uma combinação de alta temperatura e baixa umidade do ar. Dentre os gases liberados podem ser destacados o gás carbônico $\left(\mathrm{CO}_{2}\right)$ e os gases traço, como metano $\left(\mathrm{CH}_{4}\right)$, monóxido de carbono $(\mathrm{CO})$ e nitroso de oxigênio $\left(\mathrm{N}_{2} \mathrm{O}\right)$ [Fearnside 2002]. A ideia da detecção de incêndios baseada em redes de sensores é usar sensores para coletar dados como fumaça, temperatura, umidade e gases em uma floresta e criar um sistema inteligente que analise esses dados e possa alertar às equipes de combate a incêndio, reduzindo o tempo de resposta e evitando maiores perdas.

As redes Low-Power Wide Area Networks (LPWANs) utilizam comunicação sem fio para a transmissão de dados por distâncias da ordem de grandeza de quilômetros, facilitando o desenvolvimento de aplicações de cobertura de grandes áreas [Farrell 2018], como ocorre na maioria dos ambientes florestais. LoRa é uma tecnologia de rede LPWAN desenvolvida pela LoRa Alliance $^{T M}$, uma associação de mais de 500 empresas com o intuito de expandir o uso de LPWANs em aplicações de IoT [Alliance ${ }^{\mathrm{TM}} 2018$ ].

Este trabalho possui como objetivo descrever o desenvolvimento de um sistema protótipo de Internet das Coisas para detecção de incêndios florestais, o qual é composto por sensores, dispositivos de comunicação e um sistema Web de monitoramento. O trabalho apresenta os testes com sensores de temperatura, umidade, gases e chama e descreve uma análise comparativa entre os principais dispositivos de comunicação Wi-Fi e $L P W A N / L o R a$, para verificar a viabilidade de uso em um sistema real. 
O artigo está organizado conforme segue. A Seção 2 apresenta os trabalhos relacionados. A metodologia é detalhada na Seção 3. Em seguida, a Seção 4 discute os resultados do trabalho e por último, a Seção 5 enumera as conclusões e apresenta sugestões para trabalhos futuros.

\section{Trabalhos Relacionados}

A detecção precoce de incêndios florestais é um problema complexo que envolve múltiplos aspectos, entre eles: a determinação do melhor preditor [Al_Janabi et al. 2018]; o posicionamento dos sensores de forma a minimizar o número de sensores necessários para cobrir uma área Aziz et al. 2009, Fonseca et al. 2019]; o monitoramento ciente do consumo de energia [Aslan et al. 2012, Xu et al. 2018]; o aproveitamento de energia a partir do sol [U. Ganesh and Karthik 2013].

Especificamente na área de projeto e validação, a maior parte dos testes são realizados em laboratório, sem a complexidade de um ambiente real. Um exemplo é o trabalho de [Yu et al. 2005], que propõe uma rede de sensores sem fio para atuar na detecção de incêndios em tempo real, ao coletar os dados de temperatura, umidade relativa do ar e fumaça. Estes dados são enviados a um servidor, que os processa utilizando uma rede neural para determinar a probabilidade de incêndio. O resultado calculado é direcionado a um nó sorvedouro com conexão cabeada ao nó gerenciador. O nó gerenciador disponibiliza serviços aos usuários da rede, tais como leitura instantânea dos sensores e notificação de emergências. $\mathrm{O}$ trabalho ainda pode ser aplicado em outros cenários de monitoramento e detecção de variáveis.

O trabalho de [Li et al. 2006] também propõe uma rede de sensores sem fio para a detecção de incêndios florestais. As variáveis medidas pelos sensores são temperatura, umidade, pressão barométrica e intensidade de luz. Os dados lidos pelos sensores são roteados até chegar em uma estação base que possui conexão com um servidor central. O servidor possui um banco de dados para armazenamento das leituras dos sensores e hospeda uma aplicação de monitoramento da rede. O hardware utilizado em cada nó de aquisição de dados também foi construído neste trabalho. Foi anexada ao dispositivo Mica2, que possui um microcontrolador e um módulo de comunicação, uma placa com sensores selecionados para realizar a medição das variáveis. Realizou-se testes em laboratório e em áreas externas. Apesar disso, não houve implementação de uma rede de sensores em uma área florestal, sendo sugerido pelos autores como um possível trabalho futuro.

De um modo geral, esses trabalhos apresentam objetivos semelhantes. $\mathrm{O}$ trabalho desenvolvido por [Yu et al. 2005] tem seu foco voltado para o desenvolvimento de um paradigma utilizado por uma rede de sensores sem fio para detecção de incêndios florestais. Em relação ao hardware empregado na detecção, não houve desenvolvimento, sendo listados apenas possíveis tipos de sensores para medir as variáveis. O trabalho desenvolvido por [Li et al. 2006] se aproxima mais de uma solução real. Assim como o software, o hardware também foi projetado e testado.

Por outro lado, os autores de [Lazarescu 2015] discutem detalhes relevantes do projeto e o resultado da simulação e dos testes em campo de uma plataforma de IoT de baixo custo para monitoramento de incêndios florestais. Ainda que os testes de campo foram executados em uma área relativamente reduzida $(195 \mathrm{~m}$ x $45 \mathrm{~m})$, o trabalho mostrou os 
desafios em termos de robustez, custos e necessidade de manutenção devido à exposição as condições climáticas, ao tamanho da rede que aumenta a probabilidade de falhas de nós, a presença de animais, etc. O estudo sugere a escolha cuidadosa de componentes e protocolos apropriados para este tipo de aplicação, além da auto-verificação periódica e recuperação automática de erros, como parte de um projeto ciente do consumo de energia. Nesse caso, a comunicação entre as placas de sensores foi implementada usando circuitos de RF de 433-MHz, com alcance esperado de $200 \mathrm{~m}$, suficiente para cobrir a área de teste (caso contrário, um repetidor pode ser usado) conectados a gateways com GPRS.

Tomando por base as propostas dos estudos citados, foi desenvolvido um protótipo de sistema de IoT de custo reduzido para a detecção e monitoramento de incêndios florestais. O sistema abrange desde o hardware utilizado na leitura dos dados do ambiente até a exibição desses para as partes competentes em um sistema Web. Foram usados componentes comerciais e a comunicação é baseada nos protocolos Wi-Fi e LoRa com foco em baixo custo e consumo de energia. Os dispositivos selecionados para os testes foram Dragino Shield com Arduino Uno e gateway Dragino modelo LG01-P, Arduino MKR-WAN, ESP32 (com e sem LoRa) e módulos ESP8266 (Wi-Fi), que são mais acessíveis que os dispositivos Mica2 utilizados por [Li et al. 2006]. Além do Mica2 ter um custo maior que o dos dispositivos aplicados neste trabalho, não é facilmente encontrado no Brasil. Para a comunicação entre os nós e o gateway, realizou-se testes com os módulos Wi-Fi e LoRa em um parque com vegetação de cerrado. O objetivo deste trabalho foi verificar na prática a viabilidade do protótipo e a efetividade do conjunto de sensores escolhido na detecção precoce de incêndios em áreas abertas como é o caso dos parques florestais.

\section{Metodologia e Desenvolvimento do Protótipo}

A metodologia empregada neste trabalho foi teórica e prática, composta pelas etapas de: compreensão do problema e análise de soluções análogas; definição de tecnologias disponíveis para os sensores e gateways; preparação destes dispositivos; desenvolvimento do sistema Web; realização de testes em laboratório e em campo dos sensores de temperatura, de umidade e de gases, e dos módulos de comunicação Wi-Fi e LoRa.

O protótipo do sistema de IoT desenvolvido para a detecção de incêndios florestais é composto por uma rede de sensores sem fio e um sistema Web para monitoramento. Os nós da rede possuem sensores responsáveis por captar as variáveis do ambiente como temperatura, umidade, gases e chama. Os valores identificados são transmitidos dos nós para um gateway com conexão à Internet. A arquitetura do sistema é representada pela Figura 1

O fluxo de dados do sistema apresenta sentido único, isto é, inicia com a coleta dos dados pelos sensores, tem como próximo passo a transmissão de uma mensagem ao gateway, que a recebe e a envia a uma fila de mensagens interna. A mensagem é composta por um access token que identifica o nó e os dados lidos pelo sensor. Ainda, o gateway possui um processo assíncrono de consumo e envio desses dados para um servidor de aplicação Web. Esta aplicação coordena o armazenamento dos dados recebidos em um banco de dados.

Os testes dos dispositivos ocorreram nos laboratórios da instituição e posteriormente em um parque estadual, que possui vegetação de cerrado e que é palco de diversas ocorrências de incêndios, principalmente nos períodos de estiagem. Para realizar esses 


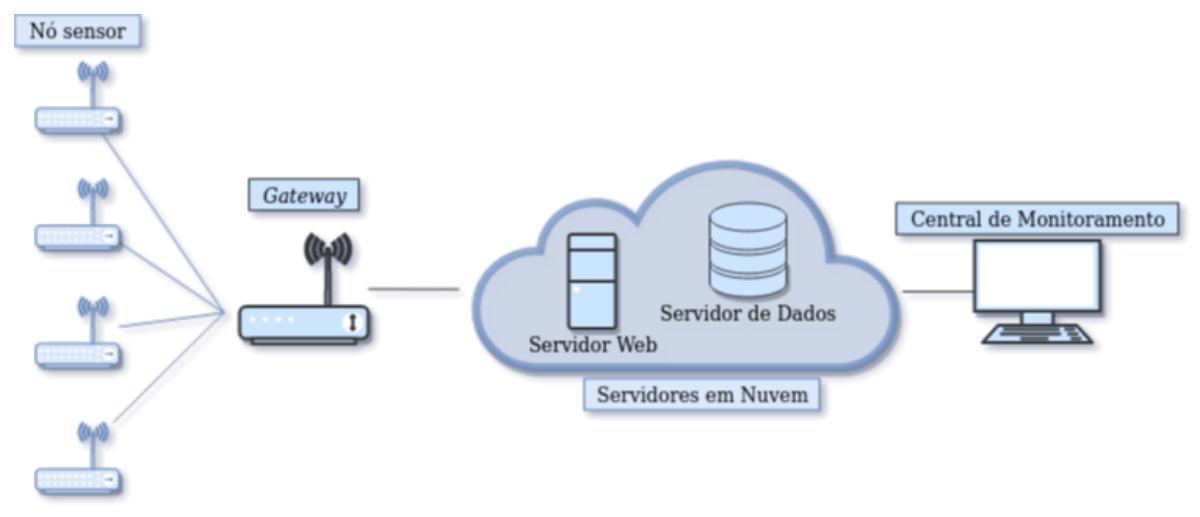

Figura 1. Diagrama ilustrativo da arquitetura do sistema.

testes aproximaram-se os kits com os sensores de temperatura/umidade, gases e de chama de uma incineração controlada por bombeiros e pela brigada de incêndio do parque. Nos kits foram utilizados os componentes apresentados na lista a seguir:

- microcontroladores Wi-Fi ESP32 Bluetooth - \$10.00;

- sensor de temperatura e umidade DHT-11 - \$5.00;

- sensor de temperatura e umidade DHT-22 - \$5.00;

- sensor de gás monóxido de carbono MQ-9 - \$7.50;

- sensor de gases inflamáveis e fumaça MQ-2 - \$6.90;

- sensor de chama FLAME-01 - \$5.95.

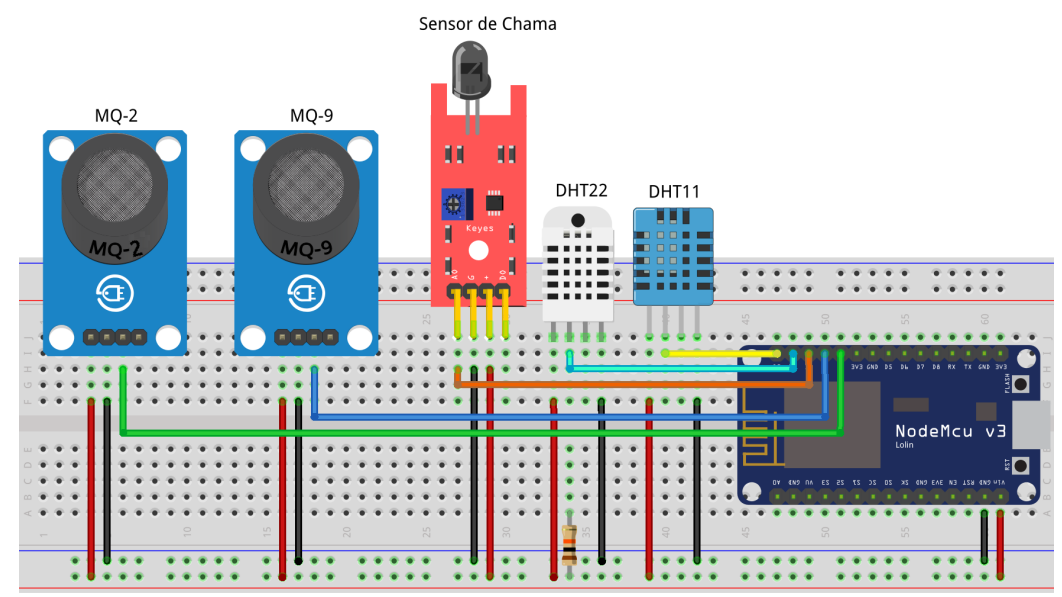

Figura 2. Esquemático ilustrativo da organização dos componentes no nó sensor.

Testaram-se 2 módulos com sensores, cada um contendo: 1 sensor DHT-11, 1 sensor DHT-22, 1 sensor MQ-2, 1 sensor MQ-9 e 1 sensor FLAME-01, conforme ilustrado na Figura 2 . Antes, durante e após a queima, realizaram-se medições com os sensores em duas diferentes distâncias, que resultaram nos gráficos de temperatura, umidade, chama, presença de gases e fumaça, apresentados na seção 4 de Testes e Resultados.

Cotação de preços dos dispositivos na distribuidora Mouser no dia 23 de Julho de 2020. 


\section{Testes e Resultados}

Os testes dos módulos de comunicação $L o R a$ e Wi-Fi consistiram na medição da distância de comunicação entre 2 módulos, um fixo e outro móvel, durante o afastamento destes. Utilizaram-se nesses testes os seguintes dispositivos:

- ESP32 LoRa $433 \mathrm{MHz}-\$ 23.00$;

- Arduino MKR WAN $1300915 \mathrm{MHz}$ - \$39.90;

- Shield Dragino $915 \mathrm{MHz}$ com Arduino Uno - \$40.90;

- Gateway Dragino LG01-P $915 \mathrm{MHz}$ - \$86.25;

- AFSmart modelos LoRa V1.1 $915 \mathrm{MHz}$ - \$51.00;

- Wi-Fi ESP8266-01 - \$6.95;

- Wi-Fi ESP8266 NodeMCU - \$8.20;

- Wi-Fi ESP8266-07s - \$10.00.

\subsection{Testes dos Sensores}

Medições captadas pelos dois kits de sensores antes, durante e depois da incineração, provocada e controlada pelos bombeiros e pela brigada de incêndio do parque, forneceram os dados para os gráficos das figuras 3 a 6 .

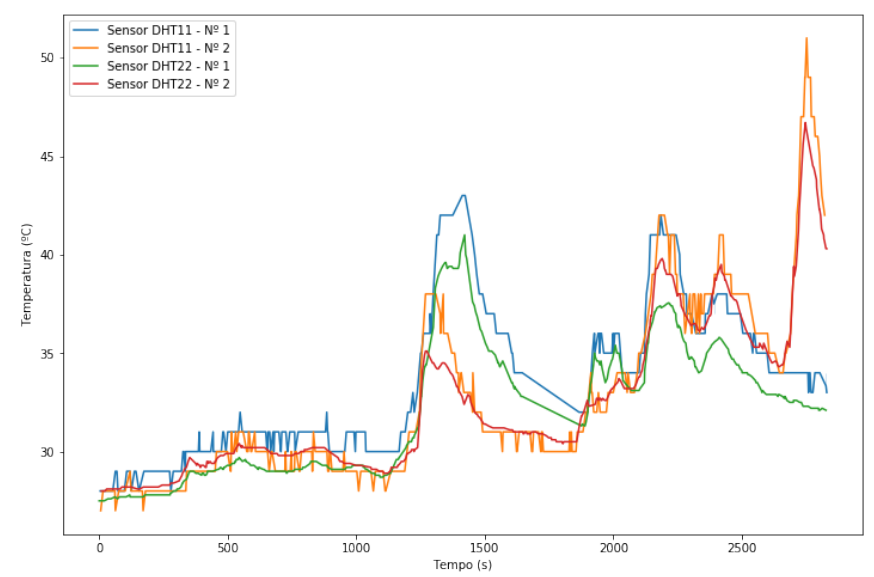

Figura 3. Leituras de temperatura dos sensores DH-11 e DHT-22.

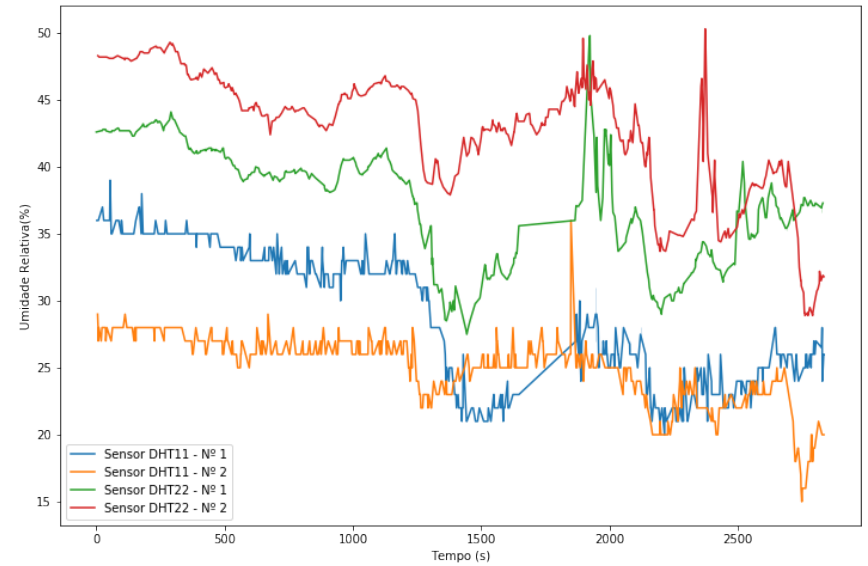

Figura 4. Leituras de umidade dos sensores DH-11 e DHT-22. 


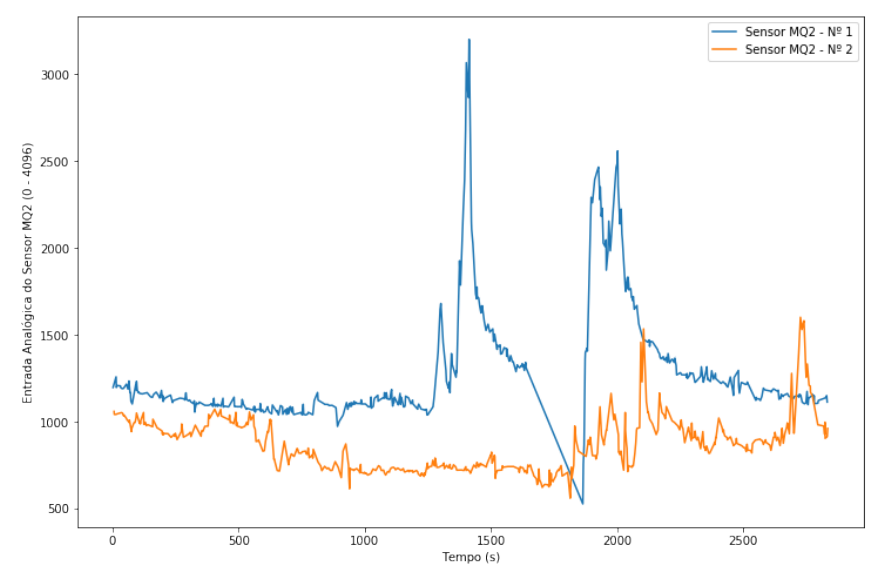

Figura 5. Leituras de fumaça dos sensores MQ-2.

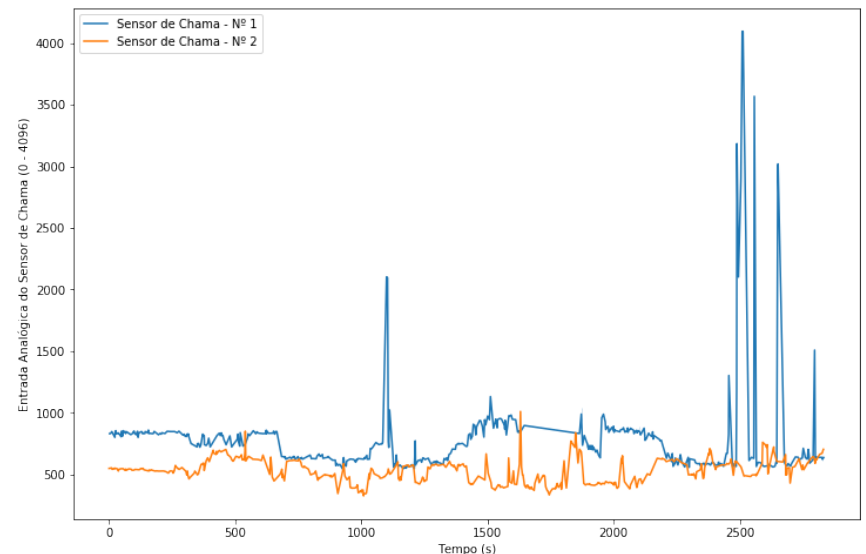

Figura 6. Leituras do sensor de chama FLAME-01.

Por meio da análise dos gráficos, obtiveram-se os cenários de detecção de incêndio. Após 20 minutos do início dos testes, ocorreu o aumento da temperatura, com variações de até $10{ }^{\circ} \mathrm{C}$. Ainda, neste mesmo instante ocorreu a queda da umidade, que entre os sensores variou de $10 \%$ a $40 \%$. Os sensores analógicos MQ-2 e FLAME-01 apresentaram valores característicos da presença de fumaça e chama, respectivamente. Por fim, constatou-se que os sensores MQ-9 dos dois módulos estavam defeituosos, não sendo possível obter dados a respeito dos gases produzidos durante o incêndio.

\subsection{Testes dos Módulos de Comunicação Wi-Fi e LoRa}

Para determinar o alcance máximo das transmissões utilizando tanto o Wi-Fi quanto o $L o R a$, fixou-se um dos dispositivos em um ambiente externo, próximo à sede da brigada de incêndio do parque, enquanto o outro era afastado gradualmente, ao longo da vegetação de cerrado. O dispositivo fixo enviava pacotes a cada segundo, e, o recebimento de pacotes pelo dispositivo móvel era observado no display LCD desse. Ao identificar o fim do recebimento de pacotes, o movimento era interrompido e a distância entre os dispositivos medida por meio de aplicativos com GPS. As Tabelas 1 e 2 apresentam as combinações de testes de comunicação e os resultados para os módulos Wi-Fi e LoRa respectivamente. 
Tabela 1. Resultados dos Testes com os Módulos Wi-Fi

\begin{tabular}{|c|c|c|c|}
\hline Frequência & Gateway & Módulo Móvel & Alcance \\
\hline $2.4 \mathrm{GHz}$ & LG01-P* & ESP 8266 ESP-01 & $60 \mathrm{~m}$ \\
\hline $2.4 \mathrm{GHz}$ & LG01-P* & ESP 8266 ESP-07 & $240 \mathrm{~m}$ \\
\hline $2.4 \mathrm{GHz}$ & LG01-P* & ESP 8266 NodeMCU & $180 \mathrm{~m}$ \\
\hline \multicolumn{4}{|c}{ * Antena sem especificação. }
\end{tabular}

Nos testes dos módulos $\mathrm{Wi}$ - $\mathrm{Fi}$, os módulos ESP-O1 obtiveram resultados razoavelmente próximos do alcance nominal (90 metros, conforme a Tabela 11), enquanto que as placas ESP-07, com antena externa alcançaram aproximadamente metade da distância nominal, 400 metros. Os módulos NodeMCU obtiveram resultados satisfatórios, ultrapassando o alcance nominal de 100 metros.

Tabela 2. Resultados dos Testes com os Módulos LoRa

\begin{tabular}{|c|c|c|c|}
\hline Frequência & Gateway & Módulo Móvel & Alcance \\
\hline $915 \mathrm{MHz}$ & LoRa Shield (2 3dB) & MKR WAN 1300* & $510 \mathrm{~m}$ \\
\hline $433 \mathrm{MHz}$ & ESP32 TTGO* & ESP32 TTGO* & $410 \mathrm{~m}$ \\
\hline $915 \mathrm{MHz}$ & MKR WAN 1300* & MKR WAN 1300* & $300 \mathrm{~m}$ \\
\hline $915 \mathrm{MHz}$ & MKR WAN 1300 (2dB) & MKR WAN 1300 (2dB) & $260 \mathrm{~m}$ \\
\hline $915 \mathrm{MHz}$ & LG01-P* & LoRa Shield* & $70 \mathrm{~m}$ \\
\hline $915 \mathrm{MHz}$ & LG01-P* & LoRa Shield (2 3dB) & $20 \mathrm{~m}$ \\
\hline \multicolumn{3}{|c|}{} & * Antena sem especificação.
\end{tabular}

Os resultados obtidos para o alcance máximo de transmissão foram de aproximadamente 500 metros para o LoRa, que transmite na faixa de frequência de $433 \mathrm{MHz}$, e de aproximadamente 250 metros para o Arduino MKR WAN 1300, que transmite na faixa de frequência de $915 \mathrm{MHz}$. Apesar de o alcance obtido ser inferior à distância na faixa de quilômetros, conforme indicado pelas especificações do padrão $L o R a$, é possível construir uma solução de comunicação de algumas centenas de metros de distância, o que representa uma resposta mais interessante para ambientes externos e que necessitam de maior cobertura do que outros padrões sem fio populares como o Wi-Fi.

\section{Conclusão}

O objetivo deste trabalho foi testar sensores e módulos de comunicação comerciais para produzir um protótipo de sistema IoT efetivo e de baixo custo para a detecção precoce de incêndios em áreas florestais.

O raio de detecção dos sensores é limitado em decorrência de características do hardware e de fatores do ambiente. Concluiu-se que, para os sensores de gases utilizados, a detecção ocorre apenas se o vento estiver incidindo sobre o sensor. A sensibilidade dos sensores de temperatura e umidade testados foi suficiente para indicar a ocorrência de incêndios ainda em curtos intervalos de tempo e a distâncias médias. Para o sensor de chama, é preciso que haja visada direta para a detecção, portanto apresenta menor eficácia em biomas densos. Cabe ressaltar, ainda, que os dados obtidos nos testes foram ruidosos e que para um sistema de tempo real é necessário um tratamento estatístico posterior. 
Os testes de alcance de comunicação, ainda em um ambiente sem obstáculos, não verificaram o alcance de alguns quilômetros como prometidos pelas especificações do padrão $L o R a$. Entretanto, o alcance da comunicação superou em algumas centenas de metros o dos módulos Wi-Fi testados. Novos estudos e testes podem ser realizados para explorar melhor a tecnologia LoRa com vistas a garantir maior alcance de comunicação.

Todavia, os testes realizados foram considerados satisfatórios, uma vez que o sistema cumpriu a função de transmissão de dados do nó para o gateway e deste para o servidor de armazenamento. Apesar de ser muito simples, a arquitetura pode servir de base para outros modelos mais robustos, atentando ao fato de que a utilização de múltiplos nós na rede pode sobrecarregar o gateway e impactar o funcionamento da rede.

Tendo em vista as restrições de alcance dos sensores mostradas neste artigo, pretende-se dar continuidade ao projeto do sistema. Através do desenvolvimento de uma metodologia que determina uma distribuição dos sensores baseada em análises de estatísticas espaciais, que realizará um monitoramento efetivo minimizando o número de kits de detecção. A partir dessa metodologia, será possível posicionar os kits de detecção na área a ser monitorada e validar o sistema.

Outro aspecto a ser considerado durante o desenvolvimento do protótipo como trabalho futuro é como maximizar a autonomia do sistema. Dependendo da densidade da floresta e altura das árvores, o uso de painéis fotovoltaicos pode-se tornar desafiador, ou até mesmo inviável. Nesse caso, outras fontes de energia podem ser consideradas.

\section{Referências}

Al_Janabi, S., Al_Shourbaji, I., and Salman, M. A. (2018). Assessing the suitability of soft computing approaches for forest fires prediction. Applied Computing and Informatics, $14(2): 214-224$.

Alkhatib, A. (2013). A review on forest fire detection techniques. International Journal of Distributed Sensor Networks, 2014.

Alkhatib, A. A. (2014). A review on forest fire detection techniques. International Journal of Distributed Sensor Networks, 10(3):597368.

Alliance $^{\mathrm{TM}}$, L. (2018). LoRa Alliance ${ }^{\mathrm{TM}}$. Disponível em: https://lora-alliance.org/. Acesso em: 19 jun. 2018.

Aslan, Y. E., Korpeoglu, I., and Özgür Ulusoy (2012). A framework for use of wireless sensor networks in forest fire detection and monitoring. Computers, Environment and Urban Systems, 36(6):614 - 625. Special Issue: Advances in Geocomputation.

Aziz, N. A. A., Aziz, K. A., and Ismail, W. Z. W. (2009). Coverage strategies for wireless sensor networks. World academy of science, Engineering and technology, 50:145-150.

Borunda, A. (2019). Climate change is contributing to California's fires. Disponível em: https://www.nationalgeographic.com/science/2019/10/climate-change-californiapower-outage/. Acesso em: 28 jun 2020.

Farrell, S. (2018). Low-Power Wide Area Network (LPWAN) Overview. RFC 8376, RFC Editor.

Fearnside, P. M. (2002). Fogo e emissão de gases de efeito estufa dos ecossistemas florestais da amazônia brasileira. Estudos Avançados, 16(44):99-123. 
Fonseca, D., Milanés, A., and Urrutia, S. (2019). Uma ferramenta para posicionamento em redes de sensores sem fio para detecção de incêndios florestais. In In: LI SIMPóSIO BRASILEIRO DE PESQUISA OPERACIONAL, 2019, Limeira. Anais eletrônicos. Campinas, Galoá, 2020. Disponivel em: ihttps://proceedings.science/sbpo2019/papers/uma-ferramenta-para-posicionamento-em-redes-de-sensores-semfio-para-deteccao-de-incendios-florestais; Acesso em: 28 jun. 2020.

G1 (2018). Incêndio mais letal da história da Califórnia deixa mais de 40 mortos; Veja números. Disponível em: https://g1.globo.com/mundo/noticia/2018/11/13/vai-a-42-ono-de-mortos-no-gigantesco-incendio-da-california.ghtml. Acesso em: $11 \mathrm{dez} .2018$.

Lazarescu, M. T. (2015). Design and Field Test of a WSN Platform Prototype for LongTerm Environmental Monitoring. Sensors, pages 9481-9518.

Li, Y., Wang, Z., and Song, Y. (2006). Wireless sensor network design for wildfire monitoring. In Intelligent Control and Automation, 2006. WCICA 2006. The sixth world congress on, volume 1, pages 109-113. IEEE.

U. Ganesh, M. Anand, S. A. M. D. P. G. and Karthik, R. (2013). Forest fire detection using optimized solar-powered zigbee wireless sensor networks. In International Journal of Scientific and Engineering Research, volume 4, page 586-596.

Xu, Y.-H., Sun, Q.-Y., and Xiao, Y.-T. (2018). An environmentally aware scheme of wireless sensor networks for forest fire monitoring and detection. Future Internet, 10(10):102.

Yu, L., Wang, N., and Meng, X. (2005). Real-time forest fire detection with wireless sensor networks. In Wireless Communications, Networking and Mobile Computing, 2005. Proceedings. 2005 International Conference on, volume 2, pages 1214-1217. IEEE.

Zhang, K., Molina-Pico, A., Cuesta-Frau, D., Araujo, A., Alejandre, J., and Rozas, A. (2016). Forest monitoring and wildland early fire detection by a hierarchical wireless sensor network. Journal of Sensors, 2016. 